Präv Gesundheitsf 2021 · 16:282-289

https://doi.org/10.1007/s11553-020-00810-z

Eingegangen: 5. Mai 2020

Angenommen: 1. August 2020

Online publiziert: 26. August 2020

(c) Der/die Autor(en) 2020

\author{
Mathias Krisam ${ }^{1,2} \cdot$ Mona Maier $^{2} \cdot$ Johannes Krisam $^{3}$ \\ 'Institut für Medizinische Soziologie und Rehabilitationswissenschaften, Charité Universitätsmedizin \\ Berlin, Berlin, Deutschland \\ ${ }^{2}$ Behavioural Science, Läuft GmbH, Berlin, Deutschland \\ ${ }^{3}$ Institut für Medizinische Biometrie und Informatik, Universität Heidelberg, Heidelberg, Deutschland
}

\title{
\#treppegehtimmer: die effektive und niedrigschwellige Möglichkeit zur Steigerung körperlicher Aktivität im Alltag
}

\section{Einleitung}

Körperliche Inaktivität ist nach Bluthochdruck, Rauchen und hohem Blutzucker der vierthäufigste Risikofaktor für Todesfälle weltweit. Trotzdem steigen körperliche Inaktivitätslevel in vielen Ländern [27]. Die WHO empfiehlt mindestens 150 min moderates Gehen pro Woche [28], aber viele Menschen erreichen nicht einmal dieses Ziel. In Europa verfehlen fast $40 \%$ dieses Ziel, daher gibt es viel Raum zur Verbesserung, gerade bei besonders inaktiven Untergruppen [13].

Die allgemeine Steigerung körperlicher Aktivität bei der Bevölkerung ist als Priorität für die öffentliche Gesundheit anerkannt. Schon die Einbeziehung körperlicher Aktivität in den normalen Tagesablauf anhand von Lifestyle-Aktivitäten kann zu einer relevanten Steigerung des Aktivitätslevel führen [22]. Dies ist im Vergleich zu umfassenden Sportprogrammen möglicherweise auch praktikabler, da eine Förderung von Sportaktivitäten oft zusätzliche Zeit, Kosten oder Zugang zu passenden Räumlichkeiten mit sich trägt und dadurch schwerer durchführbar ist, besonders auf der Bevölkerungsebene [21].

Treppensteigen ist eine der Aktivitäten, die sich für die meisten Bevölkerungsgruppen leicht in den Alltag integrieren lässt und dadurch zur Steigerung der körperlichen Aktivität im Laufe des Tages beitragen kann [2]. Boreham et al. fanden heraus, dass Treppensteigen wichtige kardiovaskuläre Risikofaktoren günstig verändert [4]. Darüber hinaus führte eine 12-wöchige Kampagne für die Treppenbenutzung an einem Arbeitsplatz zu einem Anstieg der Treppenbenutzung mit einer signifikanten $\mathrm{Zu}$ nahme der geschätzten maximalen aeroben Kapazität der Teilnehmer und einer Abnahme des Taillenumfangs, Gewichts, Fettmasse, Blutdrucks und Blutfette [14].

Studien, welche die Treppennutzung untersuchen, haben gezeigt, dass an Arbeitsplätzen die Treppenbenutzung zwischen 11,1 und 69,0\% liegt und in öffentlichen Bereichen zwischen 30,7\% bzw. 37,4\% [2]. Bisherige Studien haben verschiedene Treppeninterventionen getestet: Änderungen des sozialen Umfelds durch Aktionstage [10, 19], versendete E-Mails vom Betriebsarzt [23] und schriftliche Informationen in einer Zeitung [17] erreichten gemischte Erfolge. Vereinzelte Studien haben außerdem die Auswirkung von Vorbildern auf die Verwendung von Treppen untersucht und eine signifikante Vorbildwirkung entdeckt $[1,25]$. Die bisher am meisten untersuchten Interventionen sind sog. POD(„point of decision“)-Prompts. Beispiele solcher Prompts sind motivierende oder richtungsweisende Botschaften auf Postern, Treppenbannern und Pfeile/ Fußabdrücke auf dem Boden, die die Menschen dazu motivieren, die Treppe zu verwenden [2].
Untersuchungen zu sowie Vergleiche von unterschiedlichen POD-Prompts haben gezeigt, dass es eine Reihe verschiedener Aspekte gibt, die für die Wirksamkeit relevant sind:

Es bestehen Unterschiede in der Wirksamkeit, je nachdem ob ein POD Prompt im öffentlichen Raum oder in Betrieben eingeführt wird: Im Falle von Interventionen im öffentlichen Raum steigerte sich die Rate des Treppensteigens durchschnittlich um 5,9\% des Mittelwerts, in Betrieben hingegen nur um $0,1 \%$ [6]. Dies wird so begründet, dass POD-Prompts das Treppensteigen signifikant erhöhen, wenn zwischen Treppen und Rolltreppen gewählt werden kann, es jedoch nur schwache Hinweise für gleichwertige Auswirkungen gibt, wenn es sich bei der Alternative um einen Aufzug handelt. Seitdem haben jedoch andere Studien einen höheren Anstieg des Treppensteigens bei Interventionen in Betrieben gefunden $[6,11,19]$, was darauf hindeutet, dass ein möglicher Effekt in diesem Umfeld noch weiter untersucht werden sollte.

In Bezug auf die inhaltliche Gestaltung von POD-Prompts und deren Komplexität zeigt sich, dass einfache und spezifischere komplexe Nachrichten ähnliche Auswirkungen auf das Treppensteigen haben. Die Wirksamkeit von komplexeren Nachrichten nimmt während geschäftiger Perioden mit einer größeren Anzahl an Passanten ab, wohingegen die Wirksamkeit einfacher Nachrichten 


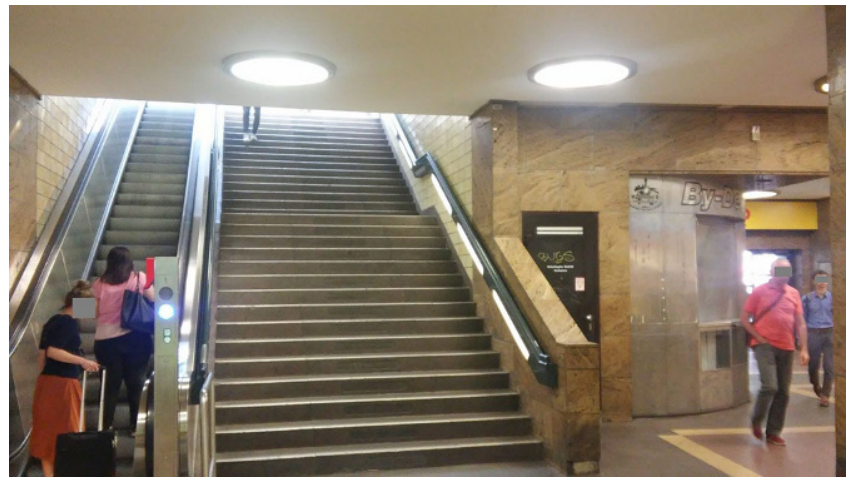

Abb. $1 \varangle$ S-Bahnhof Zoologischer Garten Eingang Hardenbergstraße

nicht vom Passantenaufkommen beeinflusst wird [12]. Die Ergebnisse legen nahe, dass einfache Nachrichten für stark frequentierte Umgebungen besser geeignet sind.

Bei der formellen Gestaltung der POD-Prompts ist unter anderem relevant, ob diese als Poster oder als Treppenstufenbanner angebracht werden. Ein systematischer Vergleich in zwei Einkaufszentren ergab, dass an den Treppenstufen angebrachte Banner das Treppensteigen doppelt so stark erhöhten wie Poster [8]. Dies kann durch größere Sichtbarkeit sowie durch das Anzeigen mehrerer Nachrichten auf Bannern (statt nur einer Nachricht auf Postern) erklärt werden [24]. Eine in einem Bahnhof durchgeführte Studie ergab jedoch, dass Banner nur in Kombination mit einem herkömmlichen Poster wirksam waren [18]. Grund dafür könnte sein, dass die Sichtbarkeit von an Treppenstufen angebrachten Bannern durch hohe Passantenzahlen beeinträchtigt wird und diese dadurch unwirksam werden. Gerade an Bahnhöfen, wo Passanten oft in größeren Gruppen zur Ankunft oder Abfahrt eines Zuges erscheinen, kann es passieren, dass Banner allein in ihrer Wirksamkeit eingeschränkt sind und eine Ergänzung durch Poster ratsam ist.

Ein anderer Aspekt der formellen $\mathrm{Ge}$ staltung, der sich auf die Wirksamkeit von POD-Prompts auswirkt, ist die Größe des Posters. Dieses sollte größer als A3 sein, um die gewünschten Effekte zu erreichen [9]. Mittelgroße (A1-A0) bis große (>A0) Poster erzielen die größte Wirksamkeit [7].

Über die möglichen Unterschiede je nach Farben der POD-Prompts sowie deren genaue positionelle Platzierung
(Entfernung zur Treppe von Postern und Richtungsschildern) wurden bisher, soweit den Forschern bekannt, noch keine Studien durchgeführt. In vielen Studien wurden bunte POD-Prompts verwendet, mit der Begründung, dass diese für eine gute Sichtbarkeit sorgen $[3,20]$.

Abgesehen von der rein formellen $\mathrm{Ge}$ staltung der POD-Prompts wurde außerdem in einer systematischen Übersichtsarbeit von Treppenstudien untersucht, wie Schilder mit motivierenden Inhalten (wie z. B. Poster und Banner, die über die Gesundheit oder den Gewichtsverlust beim Treppensteigen informieren) im Vergleich zu zusätzlichen Richtungsschildern (wie z. B. Pfeile oder Fußabdrücke, die zu Treppen hinführen) abschneiden. Studien, bei denen eine Kombination von Motivations- und Richtungszeichen verwendet wurde, berichteten häufiger über eine Zunahme des Treppensteigens als Studien, bei denen nur Motivationszeichen verwendet wurden [2]. Hierbei wirkt sich der thematische Inhalt der Motivationsnachrichten auch auf die Erfolgsraten aus: Interventionsmaterial, das sich auf Zeiteinsparung und Fitness bezieht, hat eine Erfolgsrate von $>80 \%$, gefolgt von Gesundheitsbotschaften (78\%), Gewicht (72\%) und Energieverbrauch (69\%; [7]).

Beim thematischen Inhalt von Treppeninterventionen lag bisher noch kein ausdrücklicher Fokus auf humorbasierten Nachrichten. Allgemein kann durch Humor die Aufmerksamkeit für Aussagen gesteigert $[15,26]$ und auch die Beliebtheit des Nachrichtenübermittlers erhöht werden [16]. Haben Gesundheitskampagnen einen humorvollen Kommunikationsstil, ist die Wahrscheinlichkeit größer, dass Menschen, die die Kampagne gesehen haben, auch anderen davon erzählen. Dadurch können nicht nur Effekte bei denen erzielt werden, die die Kampagne selbst wahrnehmen, sondern auch darüber hinaus durch konversationsbasierte Effekte [5].

Ziel dieser Pilotstudie war es daher, einen POD-Prompt zu entwickeln, der nach den bisherigen Erkenntnissen allen Kriterien der maximalen Wirksamkeit entspricht und diesen in einem öffentlichen Setting zu testen. Dieser PODPrompt soll außerdem nach dem ersten Test, wenn nötig, weiterentwickelt und dann in einer größeren Kampagne ausgedehnt werden, welche flächendeckend und effektiv zum Treppensteigen einlädt.

\section{Methodik}

\section{Studiendesign}

Ein zweiarmiges Prä-Post-Studiendesign mit Kontrollgruppe wurde verwendet, um die Wirksamkeit der Intervention zu testen. Zusätzlich zur Interventionstreppe wurden Messungen an einer Kontrolltreppe durchgeführt, an der keine Veränderungen vorgenommen wurden. Die Anzahl der Treppengänger und Rolltreppenfahrer wurde an beiden Treppen vor der Intervention, während der Intervention, sowie nach der Intervention gemessen.

\section{Setting}

Für das Setting wurde der öffentliche Bereich gewählt. Grund dafür war v. a., dass hierdurch eine größere soziodemographische Gruppe angesprochen und dadurch der Effekt des POD-Prompts an einer breiteren Gruppe untersucht werden kann. Des Weiteren ist das Langzeitziel, nach erfolgreichem Test und eventuellen Anpassungen, den POD-Prompt in einer flächendeckenden Kampagne zu verbreiten, was im öffentlichen Bereich geschehen soll. Daher ist das Testen in genau diesem Bereich von besonderer Wichtigkeit.

Als Setting wählten wir den Berliner S-Bahn-Bahnhof Zoologischer Garten. Dieser S-Bahnhof wird laut der Agentur Ströer täglich von ca. 140.000 Passan- 
Präv Gesundheitsf 2021 · 16:282-289 https://doi.org/10.1007/s11553-020-00810-z

(c) Der/die Autor(en) 2020

\section{Krisam · M. Maier · J. Krisam}

\section{\#treppegehtimmer: die effektive und niedrigschwellige Möglichkeit zur Steigerung körperlicher Aktivität im Alltag}

\section{Zusammenfassung}

Hintergrund. Viele Deutsche bewegen sich im Alltag zu wenig, obwohl es ausreichend Möglichkeiten gibt, mehr Bewegung einfach in den Alltag zu integrieren. Gerade Treppen werden häufig "links" liegen gelassen und die bequemere Variante in Form des Fahrstuhls oder der Rolltreppe bevorzugt. Sogenannte Treppen-Nudges bieten eine gute Möglichkeit, Menschen zur Benutzung der Treppe zu motivieren.

Zielstellung. Ziel war es daher, in einer Pilotstudie einen effektiven und kosteneffizienten Treppen-Nudge zu entwickeln, zu erproben und im Rahmen einer Ex-Post- und Kontrollstudie zu evaluieren.

Methodik. Unter dem Slogan \#treppegehtimmer wurden humorvolle Sprüche und attraktive Grafiken, die zum Treppensteigen motivierten, vor und an einer Treppe des Berliner S-Bahnhofs Zoologischer Garten im September/Oktober 2019 für 4 Wochen angebracht. Sowohl an der Interventionsals auch einer Kontrolltreppe, die ebenfalls zur Plattform führte, wurden die Anzahl der Treppengänger*innen sowie der Rolltreppenfahrer*innen vor, während und nach der Intervention, jeweils morgens (8-10 Uhr) und abends (16-18 Uhr) an ausschließlich 2 Wochentagen gemessen.

Ergebnisse. Durch die Intervention wurde die Rate der Treppengänger*innen im Vergleich zu Rolltreppenfahrer*innen signifikant gesteigert: der Kurzzeiteffekt der Intervention liegt bei einer Odds Ratio (OR) von $\mathrm{OR}=1,4305$ $(p<0,0001)$ und der Langzeiteffekt bei $\mathrm{OR}=1,4334(p<0,0001)$. Dies bedeutet, dass durch die Intervention umgerechnet täglich etwa 1200 mehr Menschen die Treppe genommen haben.

Schlussfolgerung. Diese Pilotstudie zeigt, dass \#treppegehtimmer nachweislich zu einer Steigerung der Treppengänger*innen führt und damit eine kostengünstige und effektive Möglichkeit zur Förderung von mehr Bewegung im Alltag darstellt. Die Aktion sollte daher ausgeweitet und weiter evaluiert werden.

\section{Schlüsselwörter}

Bewegungsförderung · Gesundheitsförderung · "Nudging" · Prävention . Treppensteigen - Verhaltensökonomie

\section{\#treppegehtimmer: An effective and simple way to increase physical activity in everyday life}

\section{Abstract}

Background. Many Germans do not move enough in their everyday life, despite there being enough opportunities to simply integrate more physical activity into their daily routine. Particularly taking the stairs is often disregarded and people prefer the more comfortable alternative of an elevator or escalator. So-called stair nudges are a great way to motivate people to use the stairs. Objectives. The aim of this study was therefore to develop an effective and inexpensive stair nudge, to test it in a pilot study, and to evaluate it as part of an ex-post and control study.

Methods. Under the slogan \#treppegehtimmer (\#stairsalwaysgo), humorous slogans and attractive graphics that motivate people to climb stairs were placed in front of and on a staircase of the Berlin city train station Zoologischer Garten in September/October 2019 for 4 weeks. On both the intervention and control stairs, which led to the same platform, the number of people taking the stairs and escalator before, during and after the intervention was counted in the morning (8-10 a.m.) and afternoon (4-6 p.m.) on 2 days of the week.

Results. The intervention significantly increased the rate of stair climbers compared to people taking the escalator: the short-term effect of the intervention had an odds ratio (OR) of $1.4305(p<0.0001)$ and the long-term effect had an OR of $1.4334(p<0.0001)$. This means that around 1200 more people took the stairs every day as a result of the intervention. Conclusion. This pilot study shows that \#treppegehtimmer demonstrably leads to an increase in people taking the stairs and thus represents an inexpensive and effective way to promote more physical activity in everyday life. This initiative should therefore be expanded and further evaluated.

\section{Keywords}

Physical activity promotion - Health promotion - Prevention - Nudging · Stair climbing . Behavioural economics ten frequentiert, was der Untersuchung eine große Untersuchungsgruppe ermöglichte. Es gibt genau zwei Treppen, die auf die Plattform des S-Bahnhofs hinaufführen. Als Interventionsstandort wählten wir die Treppe vom Aufgang Hardenbergstraße. Hier befindet sich eine Rolltreppe direkt neben dem Treppenaufgang (• Abb. 1, sowie Querschnitt in - Abb. 2).

Die Passanten betreten den Innenbereich des S-Bahnhofs von der Hardenbergstraße aus, und müssen, um auf die
Treppe oder Rolltreppe zu gelangen, eine $180^{\circ}$-Drehung einlegen. - Abb. 1 zeigt den Eingang von der Hardenbergstraße (rechte Bildseite) und die Rolltreppe (linke Bildseite). Die Treppe besteht aus 46 Stufen, die in drei Abschnitte mit zwei Zwischenplattformen nach 15 und 30 Stufen unterteilt sind.

Die Kontrolltreppe befindet sich im gleichen S-Bahnhof und führt Passanten von einem anderen Eingang zum selben Gleis wie die Interventionstreppe. Bei der Kontrolltreppe sind Rolltreppe und Treppe nicht direkt nebeneinander. Die Aufgänge sind durch einige Meter voneinander getrennt, jedoch sind beide Aufgänge sehr deutlich ausgeschildert. Wir wählten den Aufgang Hardenbergstraße, da hier durch die unmittelbare Positionierung von Treppe neben Rolltreppe der POD klarer zu bestimmen und dementsprechend die Steuerung durch den Prompt deutlicher und die Messung exakter wurde. 


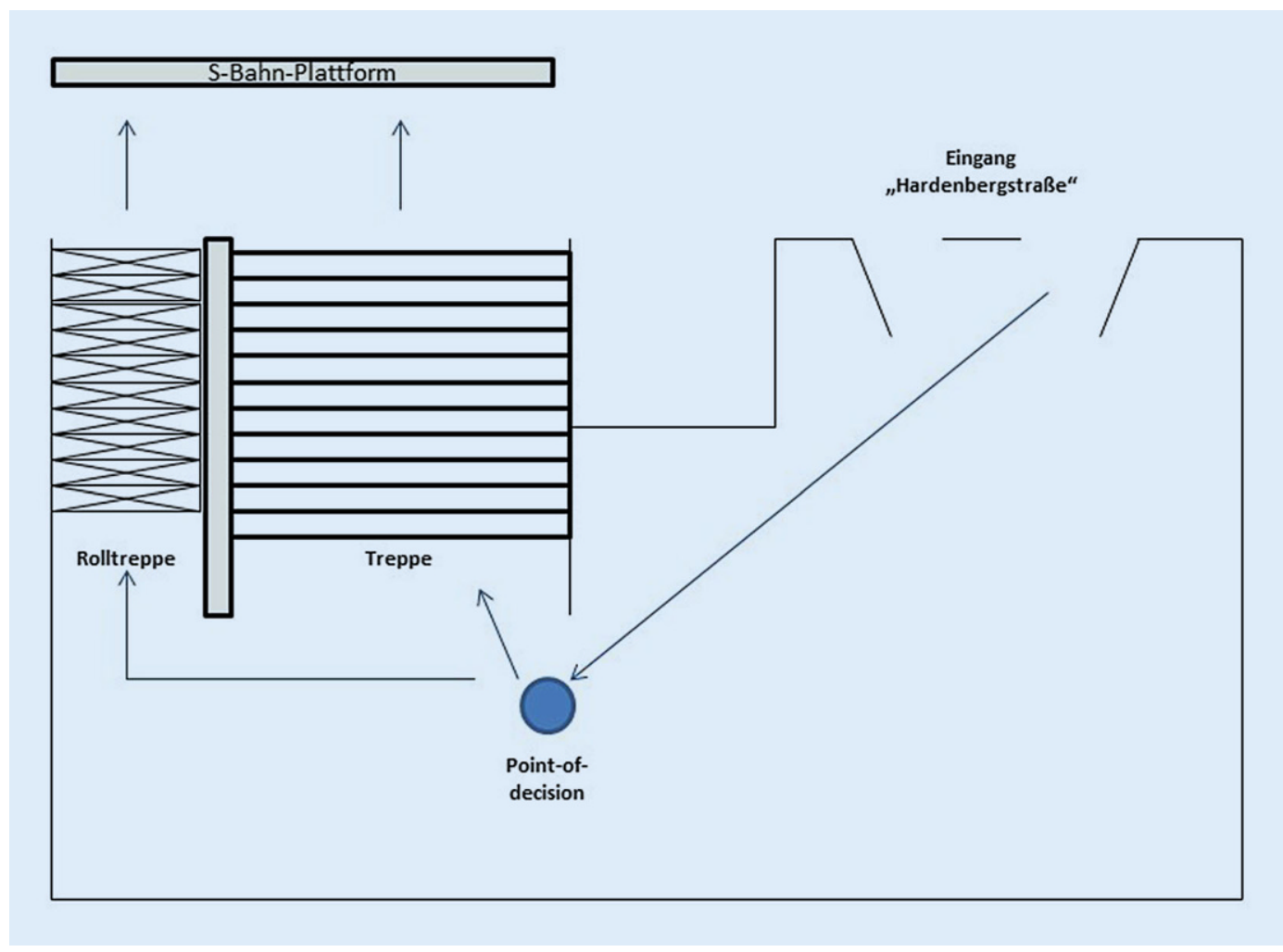

Abb. $2 \triangleleft$ Querschnitt des Settings (schematisch)
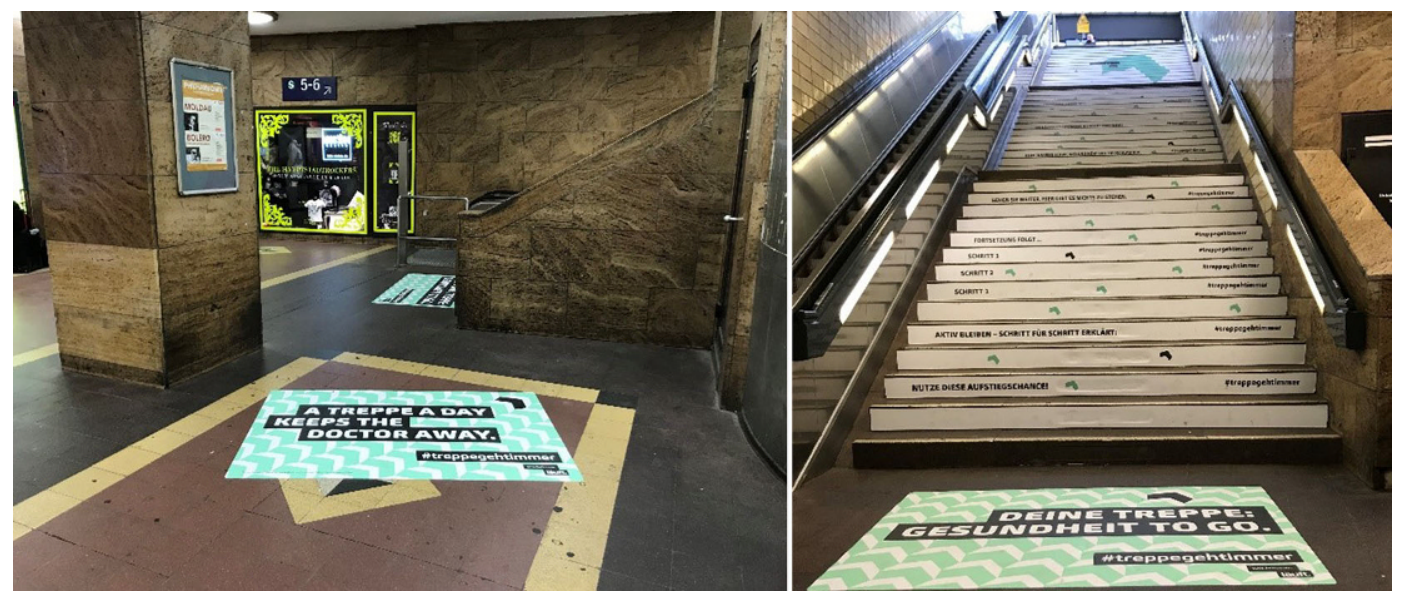

Abb. $3<$ Sprüche an Treppe

\section{Entwicklung des POD-Prompts}

Die in der Einleitung identifizierten wissenschaftlichen Erkenntnisse bildeten die Grundlage für die Entwicklung der PODPrompts. Diese wurden gemeinsam mit einer Berliner Werbeagentur entwickelt und erfüllten folgende Kriterien:

\section{Design}

1. Kombination aus Poster (am Boden vor der Treppe angebracht) und Treppenstufenbanner, um Sichtbar- keit trotz hoher Passantenzahl zu gewährleisten (• Abb. 3),

2. Bodenposter mit Mindestgröße von DIN A1 $(59,4 \times 84,1 \mathrm{~cm})$,

3. Gerade die Poster vor der Treppe sollten farblich sehr auffallend sein, um als Prompt dem Passanten ins Auge zu springen,

4. Verwendung wiederkehrender grafischer Elemente, die eine Wiedererkennung schaffen,

5. Kombination aus richtungsweisenden Elementen (Pfeile) und motivierenden Elementen (Text),
6. Grafische richtungsweisende Elemente sollten zur Treppe und von der Rolltreppe wegführen.

Text

1. Alle Nachrichten sollten so einfach und spezifisch wie möglich formuliert werden,

2. Alle Nachrichten sollten eine positive Konnotation beinhalten,

3. Vermeiden diskriminierender oder beleidigender Botschaften gegenüber 
Tab. 1 Auswahl der Sprüche

Vor der Treppe als Bodenposter $(180 \times 120 \mathrm{~cm})$

Auf den Treppenstufenbannern (von unten nach oben)
A Treppe A Day Keeps the Doctor Away

Deine Treppe: Gesundheit-To-Go

Nutze diese Aufstiegschance

Aktiv bleiben Schritt für Schritt erklärt:

Schritt 1

Schritt 2

Schritt 3

Fortsetzung folgt ...

Gehen Sie weiter, hier gibt es nichts zu stehen

Beim inneren Schweinehund hört der Tierschutz auf

Die Hälfte ist geschafft. Du noch lange nicht

Leider steil, leider geil!

Ohne Fleiß kein Gleis

Sie haben Ihr Ziel erreicht!
Tab. 2 Logistische Regressionsanalyse für die Auswahl von Treppe/Rolltreppe an einer S-BahnStation in Berlin (September bis Oktober 2019)

\begin{tabular}{|c|c|c|c|}
\hline & Odds Ratio & $95 \%-K I$ & $p$-Wert \\
\hline Konstante & 0,4239 & 0,$3818 ; 0,4705$ & $<0,0001$ \\
\hline $\begin{array}{l}\text { Gruppe (Intervention vs. Kontrolle) - vor Interven- } \\
\text { tion }\end{array}$ & 0,7522 & 0,$6818 ; 0,8297$ & $<0,0001$ \\
\hline Interventionszeitraum (nach vs. vor) & 0,8787 & 0,$8192 ; 0,9426$ & 0,0003 \\
\hline Interventionszeitraum (während vs. vor) & 0,8350 & 0,$7760 ; 0,8985$ & $<0,0001$ \\
\hline Verkehrsaufkommen (in Personen) & 1,1707 & 1,$1197 ; 1,2240$ & $<0,0001$ \\
\hline $\begin{array}{l}\text { Gruppe (Intervention vs. Kontrolle) - nach Interven- } \\
\text { tion }\end{array}$ & 1,4334 & 1,$2795 ; 1,6059$ & $<0,0001$ \\
\hline $\begin{array}{l}\text { Gruppe (Intervention vs. Kontrolle) - während } \\
\text { Intervention }\end{array}$ & 1,4305 & 1,$2799 ; 1,5990$ & $<0,0001$ \\
\hline
\end{tabular}

Randgruppen oder Rolltreppenfahrer*innen,

4. Die Treppengänger*in sollte während des Aufgangs durch humorvolle Sprüche „unterhalten“ werden, um eine positive Assoziation mit dem Treppenaufgang zu verbinden und die Aufmerksamkeit für den PODPrompt zu steigern,

5. Die „Story“ der Sprüche sollte vor der Treppe eine klare schriftliche Aufforderung zur Treppenbenutzung geben,

6. Am Ende der Treppe sollten „belohnende "Nachrichten für die Treppengänger ${ }^{*}$ in angebracht werden.

Folgende Sprüche wurden für die Interventionstreppe kreiert und ausgewählt (- Tab. 1):

\section{Intervention}

Alle Treppenaufkleber wurden am 17. September 2019 komplett vor bzw. auf der Treppe angebracht. Die Aufkleber hingen bis zum 15. Oktober 2019, also insgesamt 4 Wochen.

\section{Messung}

Die Messungen erfolgten in der Woche vor der Anbringung der Plakate, während der 4 Wochen als auch die 2 Wochen nach Entfernen der Plakate sowohl an der Interventions- als auch der Kontrolltreppe.

Die Messungen erfolgten immer für jeweils $1 \mathrm{~h}$ an Interventions- und Kontrolltreppe im Zeitraum zwischen 8 und 10 Uhr (morgendliche Messung), als auch zwischen 16 und 18 Uhr (abendliche Messung), ausschließlich montags und mittwochs. Das Zählen erfolgte anhand von analogen Handzählern (• Abb. 4).
Die Zähler*innen positionierten sich an Orten, die jeweils eine freie Sicht auf Interventions- und Kontrolltreppe garantierten.

Gezählt wurden alle Passanten, die auf den beiden Treppen jeweils entweder die Treppe oder die Rolltreppe zum Weg auf die Plattform verwendeten. Es wurden nur die Personen gezählt, die selbständig über die Treppe oder Rolltreppe auf die Plattform gelangten. Dies bedeutet, dass z. B. Kinder, die getragen oder im Kinderwagen transportiert wurden, nicht zählten. Kinder wiederum, die auf ihren eigenen Beinen, jedoch an der Hand nach oben gelangten, wurden gezählt.

\section{Statistische Analyse}

Zur Untersuchung des Effekts der Intervention wurde ein logistisches Regressionsmodell mit der abhängigen Variable („Treppe vs. Rolltreppe“) und der unabhängigen Variablen Gruppe („Intervention vs. Kontrolle"), Interventionszeitraum („während“ und „nach“ vs. „vorher"), sowie deren Interaktion, zusammen mit dem Verkehrsaufkommen (in Personen, auf Tausender-Werte standardisiert) gefittet.

Ziel war es die Nullhypothesen - $\mathrm{H}_{0}$ Kurzzeit: „Das Odds Ratio für die

Treppennutzung versus die Rolltreppennutzung ist während der Interventionsphase $=1$ ",

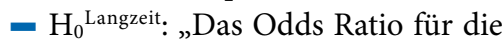

Treppennutzung versus die Rolltreppennutzung ist auch nach der Interventionsphase $=1$ “

ablehnen zu können. Um den multiplen Fehler 1. Art zu einem globalen zweiseitigen Signifikanzniveau von $5 \% \mathrm{zu}$ kontrollieren, wurde eine BonferroniKorrektur der Signifikanzniveaus für die Elementarhypothesen $\mathrm{H}_{0}{ }^{\text {Kurzzeit }}$ und $\mathrm{H}_{0}$ Langzeit vorgenommen, welche jeweils $\mathrm{zu}$ einem zweiseitigen Signifikanzniveau von $\alpha=2,5 \%$ getestet wurden. Für die OR wurden zudem zweiseitige 95\%Konfidenzintervalle (-KI) berechnet.

Während die logistische Regression die Unabhängigkeit zwischen den Beobachtungen voraussetzt, werden in Bahnhöfen einige Personen mehr als einmal beobachtet. Aufgrund des Aus- 


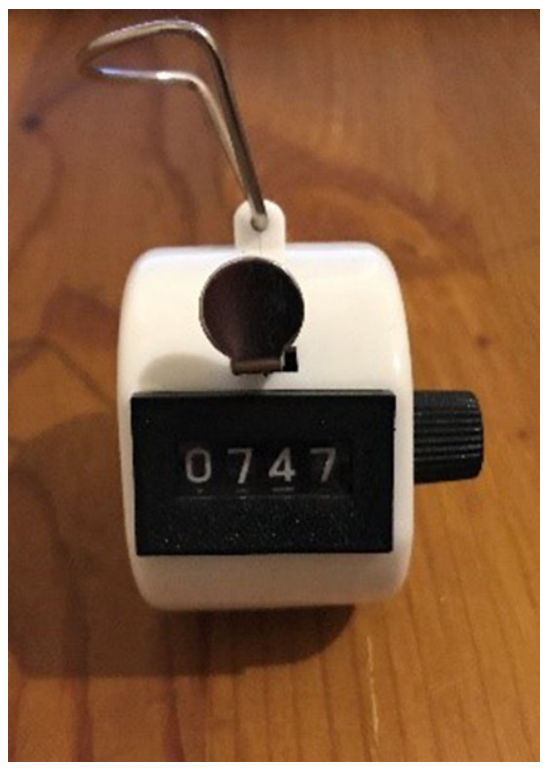

Abb. 4 \ Handzähler

maßes der Auswirkungen ist es jedoch unwahrscheinlich, dass dieser potenzielle Verstoß die Ergebnisse verändert. Die Analyse erfolgte mittels der Statistiksoftware R v 3.6 (www.r-project.org).

\section{Ergebnisse}

Innerhalb von sieben Beobachtungswochen wurden insgesamt 51.615 Fußgängerentscheidungen aufgezeichnet; 15.708 an der Interventionstreppe und 35.907 an der Kontrolltreppe. Das durchschnittliche Verkehrsaufkommen an der Interventionstreppe war 908 Personen/h und an der Kontrolltreppe 2685 Personen/h.

Basierend auf den Ergebnissen der logistischen Regression (- Tab. 2) konnten beide Nullhypothesen unter Verwendung der Bonferroni-Korrektur zu einem zweiseitigen Signifikanzniveau von $\alpha=2,5 \%$ abgelehnt werden. Hierbei liegt der Kurzzeiteffekt der Intervention bei einem OR von OR=1,4305 (95\%$\mathrm{KI}=[1,2799 ; 1,5990], p<0,0001)$ und der Langzeiteffekt bei einem Odds Ratio von $\mathrm{OR}=1,4334$ (95\%-KI $=[1,2795 ; 1,6059]$, $p<0,0001)$. Das Treppensteigen war bei der Interventionsgruppe während der Intervention häufiger als zu Studienbeginn und blieb bei der Nachuntersuchung im Vergleich zu Studienbeginn erhöht.

Der Haupteffekt des Standorts (Intervention- vs. Kontrolltreppe) lässt darauf schließen, dass die Treppenauswahl an der Kontrolltreppe während der gesamten Studie signifikant höher war. Dieser Effekt ist in - Abb. 5 ersichtlich, in der der prozentuale Anteil der Treppennutzung nach Ort, Zustand und Tageszeit stratifiziert dargestellt sind.

Es gab außerdem einen typischen positiven signifikanten Effekt des Verkehrsaufkommens (OR=1,1707; $p<0,0001)$, bei welchen sich Fußgänger*innen bei erhöhtem Verkehrsaufkommen vermehrt für die Treppe entschieden. Da ein gewisser Grad an Abhängigkeit zwischen den beiden Faktoren "Standort" und „Verkehrsaufkommen“ aufgrund des insgesamt höheren Verkehrsaufkommens am Kontrollstandort herrschte, sind diese beiden Faktoren jedoch unbedingt in einem gemeinsamen Kontext zu interpretieren. Der Faktor „Verkehrsaufkommen“ misst hierbei den Einfluss des Verkehrsaufkommens unter Festhaltung des Faktors Standort, und bildet somit im Modell u.a. die erhöhte Treppennutzung durch ein unterschiedlich starkes Verkehrsaufkommen morgens und abends $\mathrm{ab}$, nicht jedoch den Einfluss des unterschiedlich hohen Verkehrsaufkommens an beiden Standorten, welches über den Faktor Standort abgebildet ist.

Wir können somit zeigen, dass die Rate der Treppennutzer*innen während der Interventionsphase um insgesamt um $14 \%$ zunahm mit einer größeren Zunahme am Morgen $(33,4 \%)$ als am Abend (10,1 \%). Legt man die laut Ströer angegebene tägliche Besucherzahl des S-Bahnhofs von 140.000 Passanten/Tag und die von uns gemessene Verteilung an den beiden Treppen zugrunde, können wir auf Basis der vorliegenden Daten kalkulieren, dass durch den Treppen-Nudge täglich etwa 1200 Personen mehr die Treppe genommen haben.

\section{Diskussion}

\section{Diskussion der Ergebnisse}

Wir konnten mit dieser Pilotstudie zeigen, dass der Treppen-Nudge \#treppegehtimmer mit humorvollen Sprüchen und einem ansprechenden Design im öffentlichen Raum für eine höhere Benutzungsrate der Treppe sorgt. Die Änderungen halten interessanterweise auch noch nach dem Entfernen der Aufkleber an. Damit können wir viele positive Ergebnisse in Bezug auf Treppen-Nudges bzw. POD-Prompts bestätigen.

Auffallend ist, dass die Steigerungsrate am Morgen höher ausfällt als am Abend. Dies muss jedoch vor dem Hintergrund betrachtet werden, dass die Treppennutzungsrate am Morgen bereits vor der Intervention 11 Prozentpunkte niedriger lag als am Abend. Unsere Erklärung ist, dass am Abend es generell durch das größere Aufkommen zu kleinen Verzögerungen aufder Rolltreppe kommt und daher vermehrt Passanten den Weg über die Treppe nehmen. Ein weiterer Grund hierfür könnte sein, dass der höhere Verkehr auch dafür sorgt, dass die Aufkleber und damit die Aufforderung zur Treppenbenutzung weniger Passanten auffallen und daher der Effekt der POD-Prompts reduziert wird.

Weiterhin ist interessant, dass die Effekte auch nach dem Entfernen der Sprüche angehalten haben. Beim anhaltenden Effekt stellt sich die Frage, ob der Anstoß, der durch die Plakate gegeben wurde, ggf. auch (langfristige) Routinen in dem Besteigen der Bahnhofsplattform verändert hat.

\section{Limitationen}

Bei dieser Studie handelt es sich lediglich um eine Pilotstudie. Für eine genauere Analyse müsste man einen längeren Zeitraum beobachten, sowie wesentlich mehr Faktoren berücksichtigen, die das Treppenverhalten beeinflussen können. Nichtsdestotrotz liefert die Untersuchung eine starke Evidenz für den flächendeckenden Einsatz von \#treppegehtimmer in Deutschland.

$\mathrm{Zu}$ weiteren Faktoren, die untersucht werden sollten, zählen Geschlecht, Alter, Tragen von Gepäck oder anderen sperrigen Geräten, Wetter etc. Eine solche Untersuchung konnte jedoch unter den vorgenommenen Messbedingungen nicht erfüllt werden. Außerdem möchten wir darauf hinweisen, dass in der vorliegende Untersuchung Interventionsund Kontrolltreppe unterschiedliche Charakteristika aufwiesen, die auf die Werte Einfluss nehmen konnten. So war das Aufkommen an der Kontrolltreppe 


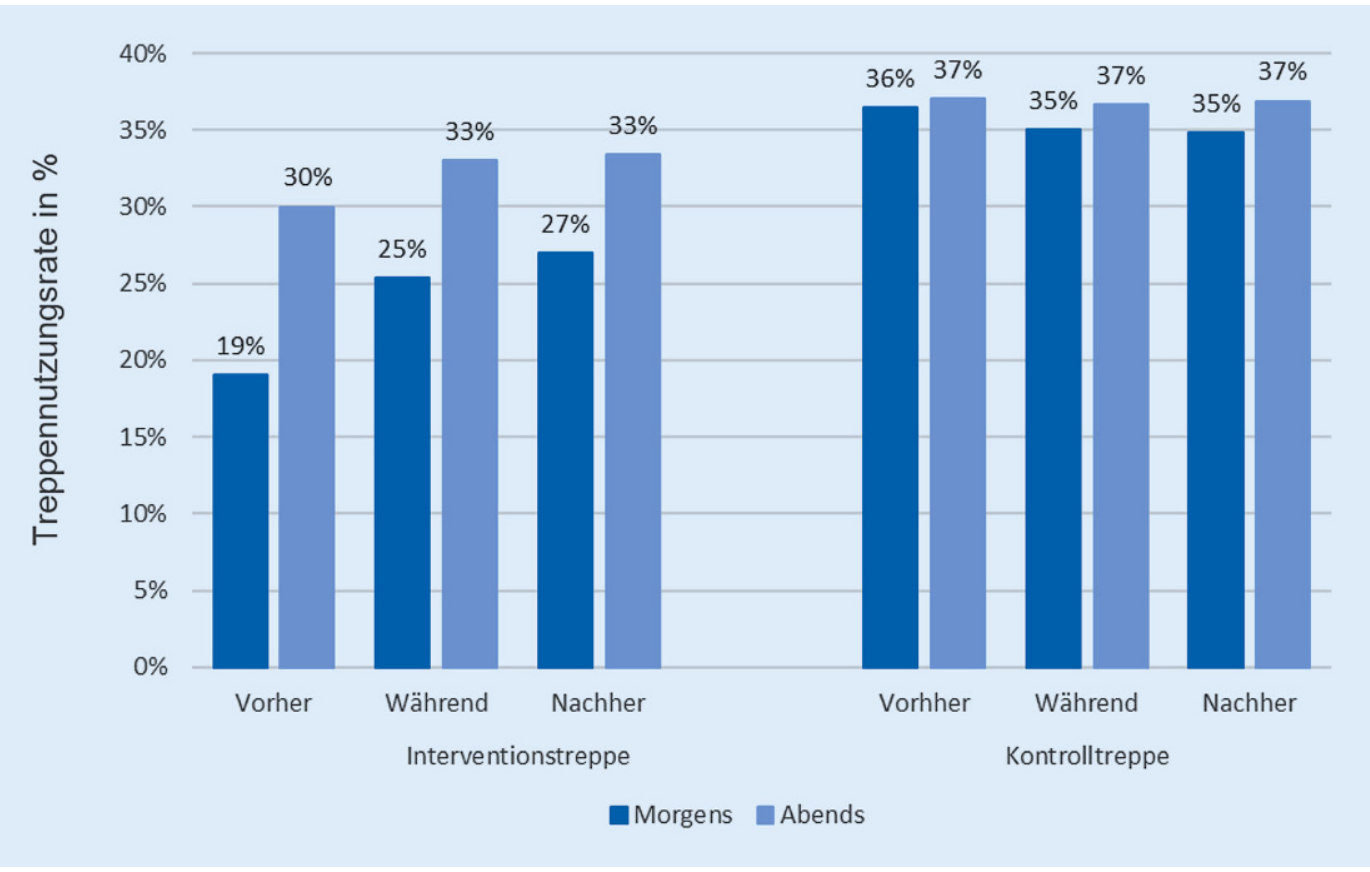

Abb. $5 \triangleleft$ Prozentualer Anteil der Treppennutzung an zwei Treppen eines Berliner S-Bahnhofs (September-Oktober 2019), stratifiziert nach Zustand und Tageszeit

in absoluten Zahlen höher als an der Interventionstreppe. Außerdem unterschieden sich auch die Baseline-Raten in Bezug auf Treppengänger und Rolltreppenfahrer. Weiterhin kann es sein, dass durch den unterschiedlichen Treppen- bzw. Rolltreppenaufgang sich der Entscheidungsprozess je Treppe stark voneinander unterscheidet. Eine Folgeuntersuchung könnte den Effekt der POD-Prompts an der nun als Kontrolltreppe verwendeten Treppe untersuchen.

\section{Anstoß für weitere Studien und offene Fragen}

Wie bereits im vorigen Abschnitt angedeutet, könnte diese Intervention in $\mathrm{Zu}$ kunft an mehreren Standorten ausgerollt und getestet werden. Dies sollte insbesondere auch Treppen mit anderem Aufgang und Treppen-/Rolltreppenplatzierung berücksichtigen. Weiterhin könnten die oben genannten Faktoren gemessen und ausgewertet werden. Hierfür wären Videoaufnahmen sehr hilfreich, welche auch gleichzeitig einen wesentlich größeren Untersuchungszeitraum und Untersuchungsgröße ermöglichen könnte. Juristische und ethische Fragen müssten diesbezüglich geklärt werden.

Genauso spannend wäre es, zu untersuchen, welche Sprüche an welcher
Position und bei welchen Zielgruppen besonders gut oder weniger gut ankommen und das gewünschte Ziel erreichen.

Weiterhin könnte eine zusätzliche qualitative Erhebung Erkenntnisse in Bezug auf die Rezeption bei den Passanten generieren: Haben sich z.B. veränderte Routinen der Treppenbenutzung eingestellt?

\section{Fazit für die Praxis}

- \#treppegehtimmer als sog. TreppenNudge ist eine einfache, kostengünstige und niedrigschwellige Möglichkeit, um flächendeckend für mehr Bewegung im Alltag zu sorgen.

- Es stellt damit eine gute Möglichkeit dar, im öffentlichen und privaten Raum effiziente Gesundheitsförderung zu betreiben.

- Die Wirkung dieses Treppen-Nudges sollte in weiteren größeren Studien mit der Möglichkeit zu noch differenzierteren Aussagen erprobt werden.

\section{Korrespondenzadresse}

\section{Dr. Mathias Krisam}

Institut für Medizinische Soziologie und Rehabilitationswissenschaften, Charité Universitätsmedizin Berlin

Charitéplatz 1, 10117 Berlin, Deutschland

mathias.krisam@charite.de

Funding. Open Access funding provided by Projekt DEAL.

\section{Einhaltung ethischer Richtlinien}

Interessenkonflikt. M. Krisam und M. Maier arbeiten für die Firma läuft $\mathrm{GmbH}$, die den Treppen-Nudge entwickelt hat und Dienstleistungen im Kontext von Verhaltenswissenschaften und Gesundheit anbietet Die läuft $\mathrm{GmbH}$ hat zudem diese Aktion finanziert. J. Krisam gibt an, dass kein Interessenkonflikt besteht.

Für diesen Beitrag wurden von den Autoren keine Studien an Menschen oder Tieren durchgeführt. Für die aufgeführten Studien gelten die jeweils dort angegebenen ethischen Richtlinien. Die Autoren geben an, dass alle Daten anonym und aggregiert ausgewertet wurden. Es erfolgte keine Dokumentation persönlicher Merkmale.

Open Access Dieser Artikel wird unter der Creative Commons Namensnennung 4.0 International Lizenz veröffentlicht, welche die Nutzung, Vervielfältigung, Bearbeitung, Verbreitung und Wiedergabe in jeglichem Medium und Format erlaubt, sofern Sie den/die ursprünglichen Autor(en) und die Quelle ordnungsgemäß nennen, einen Link zur Creative Commons Lizenz beifügen und angeben, ob Änderungen vorgenommen wurden. 
Die in diesem Artikel enthaltenen Bilder und sonstiges Drittmaterial unterliegen ebenfalls der genannten Creative Commons Lizenz, sofern sich aus der Abbildungslegende nichts anderes ergibt. Sofern das betreffende Material nicht unter der genannten Creative Commons Lizenz steht und die betreffende Handlung nicht nach gesetzlichen Vorschriften erlaubt ist, ist für die oben aufgeführten Weiterverwendungen des Materials die Einwilligung des jeweiligen Rechteinhabers einzuholen.

Weitere Details zur Lizenz entnehmen Sie bitte de Lizenzinformation auf http://creativecommons.org/ licenses/by/4.0/deed.de.

\section{Literatur}

1. Adams MA, HovellMF, Irvin Vetal (2006) Promoting stair use by modeling: an experimental application of the Behavioral Ecological Model. Am J Health Promot 21(2):101-109

2. Bellicha A, Kieusseian A, Fontvieille A-Metal (2015) Stair-use interventions in worksites and public settings-a systematic review of effectiveness and external validity. Prev Med 70:3-13

3. Bellicha A, Kieusseian A, Fontvieille A-Metal (2016) A multistage controlled intervention to increase stair climbing at work: effectiveness and process evaluation. Int J Behav Nutr Phys Act 13:47

4. Boreham CAG, Kennedy RA, Murphy MH et al (2005) Training effects of short bouts of stair climbing on cardiorespiratory fitness, blood lipids, and homocysteine in sedentary young women. $\mathrm{Br}$ JSports Med 39(9):590-593

5. Campo S, Askelson NM, Spies EL et al (2013) "Wow, that was funny": the value of exposure and humor in fostering campaign message sharing. SocMark Q 19(2):84-96

6. Eves FF (2010) Point-of-decision prompts to increase stair use. Am JPrev Med 38(5):573-574

7. Jennings CA, Yun L, Loitz CC et al (2017) A systematic review of interventions to increase stair use. Am J Prev Med 52(1):106-114

8. Kerr J, Eves F, Carrol D (2001) Encouraging stair use: stair-riser banners are better than posters. Am J Public Health 91(8):1192-1193

9. Kerr J, Eves FF, Carroll D (2001) The influence of poster prompts on stair use: the effects of setting, poster size and content. Br J Health Psychol 6(Part 4):397-405

10. Lee KK, Perry AS, Wolf SA et al (2012) Promoting routine stair use: evaluating the impact of a stair prompt across buildings. Am J Prev Med 42(2):136-141

11. Lewis A, Eves F (2012) Prompt before the choice is made: effects of a stair-climbing intervention in university buildings. $\mathrm{Br} \mathrm{J}$ Health Psychol 17(3):631-643

12. Lewis A, Eves FF (2012) Prompts to increase stair climbing in stations: the effect of message complexity. JPhys Act Health 9:954-961

13. Marques A, Sarmento $H$, Martins J et al (2015) Prevalence of physical activity in European adults-Compliance with the World Health Organization's physical activity guidelines. Prev Med 81:333-338

14. Meyer P, Kayser B, Kossovsky MP et al (2010) Stairs instead of elevators at workplace: cardioprotective effects of a pragmatic intervention. Eur JCardiovasc PrevRehabil 17(5):569-575

15. Monahan JL (1995) Thinking positively: using positive affect when designing health messages.
In: Maibach E, Parrott R (Hrsg) Designing health messages. SAGE, Thousand Oaks, S81-98

16. Nabi RL, Moyer-Gusé E, Byrne S (2007) All joking aside: a serious investigation into the persuasive effect of funny social issue messages. Commun Monogr 74(1):29-54

17. Nomura T, Yoshimoto Y, Akezaki Y et al (2009) Changing behavioral patterns to promote physical activity with motivational signs. Environ Health Prev Med 14(1):20-25

18. Olander EK, Eves FF, Puig-Ribera A (2008) Promoting stair climbing: stair-riser banners are better than posters ... sometimes. Prev Med 46(4):308-310

19. OlanderEK, EvesFF (2011) Effectiveness and cost of two stair-climbing interventions-less is more. Am J Health Promot 25(4):231-236

20. Phing CH, Saad HA, Nisak MB et al (2017) Effectiveness of physical activity intervention among government employees with metabolic syndrome. J Exerc Sci Fit 15:55-62

21. Smith LP, Ng SW, Popkin BM (2014) No time for the gym? Houseworkand other non-labor market time use patterns are associated with meeting physical activity recommendations among adults in fulltime, sedentary jobs. Soc Sci Med 120:126-134

22. van Roie E, Delecluse C, Opdenacker J et al (2010) Effectiveness of a lifestyle physical activity versus a structured exercise intervention in older adults. J Aging Phys Act 18:335-352

23. Vanden Auweele $Y$, Boen F, Schapendonk $W$ et al (2005) Promoting stair use among female employees: the effects of a health sign followed by an E-mail.J SportExerc Psychol 27(2):188-196

24. Webb OJ, Eves FF (2005) Promoting stair use: single versus multiple stair-riser messages. Am J Public Health 95(9):1543-1544

25. Webb OJ, Eves FF, Smith L (2011) Investigating behavioural mimicry in the context of stair/ escalator choice. Br J Health Psychol 16(Pt 2):373-385

26. Weinberger MG, Gulas CS (1992) The impact of humor in advertising: a review. J Advert 21(4):35-59

27. WHO (2011) New physical activity recommendations for reducing disease and prevent deaths. https://www.who.int/chp/media/news/releases/ 2011_2_physicalactivity/en/. Zugegriffen: 14. Nov. 2019

28. WHO (2018) Physical activity. https://www. who.int/news-room/fact-sheets/detail/physicalactivity.Zugegriffen:14. Nov. 2019 